\title{
X-Linked Candidate Genes for a Ciliopathy-Like Disorder
}

\author{
Ashleigh R. Pavey ${ }^{a-c}$ Thierry Vilboux ${ }^{c}$ Holly E. Babcock ${ }^{d, g}$ Margot Ahronovich $^{e}$ \\ Benjamin D. Solomon ${ }^{c, d}, f$
}

${ }^{a}$ Department of Pediatrics, Walter Reed National Military Medical Center and b Department of Pediatrics, Uniformed Services University of Health Sciences, Bethesda, Md., 'Division of Medical Genomics, Inova Translational Medicine Institute, and d Department of Pediatrics and eFairfax Neonatal Associates, Inova Children's Hospital, Inova Health System, Falls Church, Va., ${ }^{f}$ Department of Pediatrics, Virginia Commonwealth University School of Medicine, Richmond, Va., and 9 Division of Genetics and Metabolism, Children's National Medical Center, Washington, D.C., USA

\section{Established Facts}

- Chromosomal microarray and next-generation sequencing technologies are useful tools in identifying novel mutations related to genetic conditions.

- Over 1,000 cilia-associated proteins crucial to a wide range of biological functions have been identified; however, the genes encoding these proteins have only been identified in a small subset.

- Alterations in the structure or function of primary cilia can lead to a number of conditions with overlapping clinical features such as distinctive facial dysmorphisms, polycystic kidneys, and skeletal anomalies.

\section{Novel Insights}

- A male patient, with a large maternally inherited X chromosome deletion including 4 genes of poorly known biological function, was found to have clinical features resembling a ciliopathy phenotype several of these genes are strong novel candidates for ciliopathies and would bear testing in larger cohorts.

\section{Key Words}

Chromosomal microarray · Ciliopathy · Deletion · Multiple congenital anomalies $\cdot \mathrm{X}$-linked inheritance

\section{Abstract \\ The ability to interrogate the genome via chromosomal mi- croarray and sequencing-based technologies has acceler- ated the ability to rapidly and accurately define etiologies as}

well as new candidate genes related to genetic conditions. We describe a male patient with a lethal presentation of a multiple congenital anomaly syndrome that appeared consistent with a ciliopathy phenotype. The patient was found to have a novel maternally inherited 1.9-Mb X chromosome deletion including 4 known genes. Presently, the biological functions of these genes are not well delineated. However, at least one of these genes may be a promising candidate

\section{KARGER}

E-Mail karger@karger.com www.karger.com/msy (c) 2016 S. Karger AG, Base

1661-8769/16/0071-0037\$39.50/0
Benjamin D. Solomon

Division of Medical Genomics, Inova Translational Medicine Institute 3300 Gallows Road, 2nd Floor, Claude Moore Building Falls Church, VA 22042 (USA)

E-Mail benjamin.solomon@inova.org 
gene for this pattern of anomalies based on the function of related genes and information from publicly available copy number variant databases of control and affected individuals. These genes would bear further scrutiny in larger cohorts of patients with similar phenotypes.

(C) 2016 S. Karger AG, Basel

Congenital anomalies, occurring overall in more than 3 in 100 liveborn infants, are associated with significant neonatal mortality and childhood disability [Lu et al., 2008]. Thorough evaluation and timely identification of anomalies is vital for providing information related to patient care, prognosis and family planning. Infants born with multiple congenital anomalies (MCA) affecting several organ systems can be diagnostically challenging despite the likelihood of an underlying genetic etiology. However, advances in genomic technology, related to detecting genetic variants ranging from alterations in chromosome structure to point mutations, are allowing for more rapid and accurate diagnosis.

Historically, laboratory genetic evaluation in infants with MCA was limited to G-banded karyotyping used alone or in combination with FISH employing chromosome or locus-specific probes [Resta and Memo, 2012]. These tools may still be appropriate for initial testing in individuals with characteristic dysmorphisms generating strong clinical suspicion for a specific chromosomal or microdeletion syndrome or a clear family history of a specific disorder. In recent years, however, chromosomal microarray (CMA) is being increasingly utilized as first-tier genetic testing in infants born with MCA [Miller et al., 2010; Resta and Memo, 2012]. CMA is particularly useful in patients without a clearly recognizable molecular diagnosis, but where the phenotype suggests a contiguous gene deletion syndrome. Next-generation sequencing, such as whole-exome sequencing (WES) and whole-genome sequencing (WGS) are emerging technologies capable of detecting structural variants within or outside the coding regions [Biesecker and Green, 2014]. CMA and WES/WGS are increasing in availability, though controversy exists regarding when and for whom these genome-wide analysis technologies should be employed. Due to the amount of data generated by these techniques related to our overall incomplete knowledge of the effects of many rare variants, there are often interpretative challenges in understanding biological and clinical significance of the results [Majewski et al., 2011; Marian, 2012]. However, recent studies show an overall diagnostic yield of up to $20 \%$ for CMA and at least
15-30\% for sequencing-based modalities [Miller et al., 2010; Volk et al., 2015].

Here, we present a patient born with MCA including distinctive facial features, polycystic kidneys and skeletal anomalies, whose CMA identified a novel maternally inherited 1.9-Mb deletion on the X chromosome, reported as a variant of unclear clinical significance. The clinical phenotype was suspicious for a ciliopathylike disorder, and clinical sequencing of 74 known ciliopathy-related genes failed to identify any pathogenic mutations. Although the deletion identified by CMA has not been described in any currently reported ciliopathies, several of the genes in the deletion interval may be considered candidate genes for this class of disorder.

\section{Case Report}

The patient was delivered via spontaneous vaginal delivery after 37 0/7 weeks of gestation to a 30-year-old G6G6P5014 woman with a prenatal course significant for MCA identified via ultrasound (U/S). Prenatal U/S revealed fetal hydrops, cystic hygroma, right pleural effusion, thoracic cyst, talipes equinovarus, and ambiguous genitalia. Karyotype via amniocentesis revealed a normal male chromosome complement $(46, \mathrm{XY})$ using routine G-banding. There were no maternal health problems reported prior to or during this pregnancy. There were no reports of infectious or chemical exposures, fevers, bleeding, or consanguinity. The mother's obstetrical history includes 4 apparently healthy, term vaginal deliveries, productive of 3 female infants and 1 male infant. She had a fetal demise of a male conceptus at 17 weeks of gestation. In the case of the fetal demise, there were no reported congenital anomalies, though neither genetic studies nor autopsy were performed. Known maternal and paternal family histories are noncontributory, although maternal family history is limited due to the mother being adopted.

At birth, the boy weighed $2,307 \mathrm{~g}$ (10-25th centile), length was $45.7 \mathrm{~cm}(10-25$ th centile) and occipitofrontal head circumference was $32 \mathrm{~cm}$ (10-25th centile). APGARs were 5 and 6 at 1 and $5 \mathrm{~min}$, respectively. On physical exam, distinctive facial features included a large anterior fontanelle with wide sutures; markedly dysplastic, low-set and posteriorly angulated ears, and microretrognathia. He had limb anomalies including right preaxial polydactyly, marked edema of the hands and feet, internally rotated lower limbs with talipes equinovarus, and bilateral vertical talus. Other findings included generalized hypotonia, ambiguous genitalia, and redundant nuchal skin with decreased elastic coil. Premortem radiographs demonstrated a bell-shaped chest and dysplastic acetabuli. Abdominal U/S showed echogenic kidneys with small cortical cysts. Pelvic U/S revealed a single, right-sided undescended testicle and no uterus. A head U/S was suggestive of a Dandy-Walker malformation (DWM). An echocardiogram revealed patency of the ductus arteriosus and foramen ovale with otherwise normal cardiac structure and function. Due to the infant's critical medical condition and rapid demise, some intended imaging studies (e.g. brain MRI) were not performed.
38

Mol Syndromol 2016;7:37-42 DOI: $10.1159 / 000444666$
Pavey/Vilboux/Babcock/Ahronovich/ Solomon 


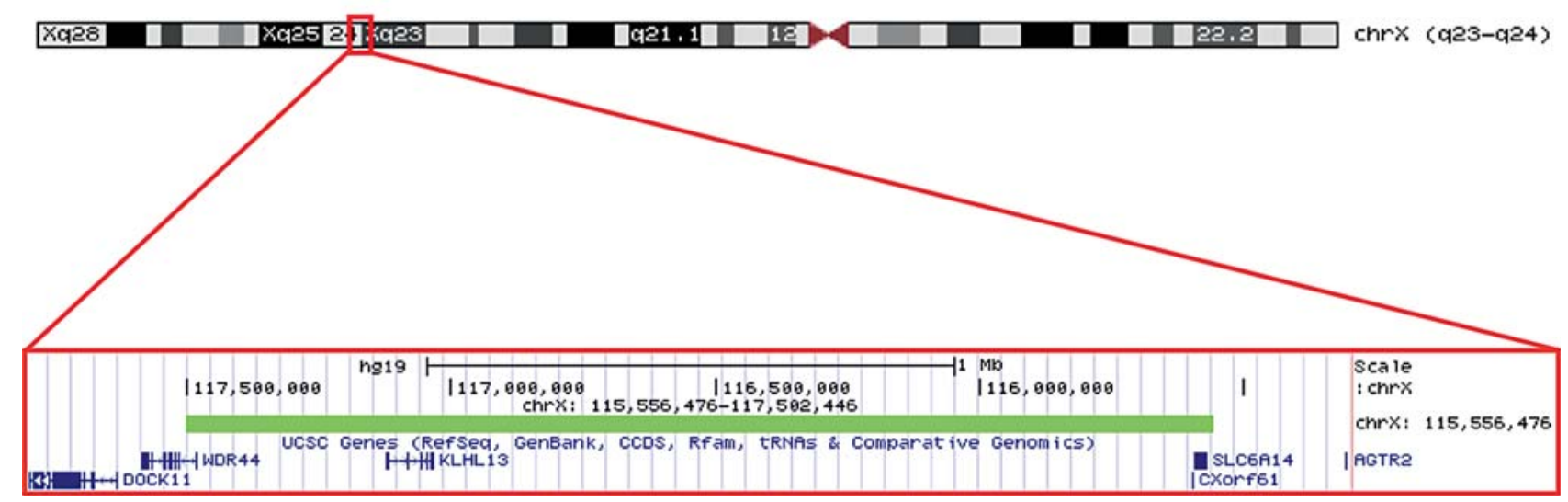

Fig. 1. Screen capture of the UCSC Human Genome Browser (http://genome.ucsc.edu) of region Xq23q24 (chrX: $115,300,000-117,800,000$ [hg19]) with the UCSC genes. The custom track (green) represents the 1.9-Mb deletion identified in the patient.

Array CGH (Quest Diagnostics, Nichols Institute, Chantilly, Va., USA) was performed and revealed an $\sim 1.9-\mathrm{Mb}$ deletion on the X chromosome: chrX: 115,556,476-117,502,446×0 [hg19] (fig 1). This interval contains SLC6A14, KLHL13, CXorf61, and a segment of the WDR44 gene. Follow-up testing revealed the same deletion in the apparently healthy mother. Sequencing of 74 ciliopathy-related genes was performed using a clinically available next-generation sequencing panel (Prevention Genetics, Marshfield, Wis., USA), which did not reveal any causative mutations. The family declined further genetic testing of siblings or other family members. Our patient's clinical condition continued to deteriorate, and he died due to respiratory failure on the 4th day of life. The family declined an autopsy or further clinical testing.

\section{Discussion}

We describe a patient with MCA who was found to have a $1.9-\mathrm{Mb}$ deletion in the $\mathrm{X}$ chromosome. Maternal testing revealed an identical deletion, suggesting X-linked recessive inheritance. The history of the previous male demise at 17 weeks of gestation is also suggestive of this inheritance pattern. The deleted region in the X chromosome contains SLC6A14, KLHL13, CXorf61, and a segment of the WDR44 gene, though little is known about their biologic functions.

X-Linked Genes for Ciliopathy-Like Disorder
Evaluation of the deleted interval using the Database of Genomic Variants (http://dgv.tcag.ca/dgv/app/home, last accessed 7/7/15) did not show large deletions overlapping the entire deleted region. There were no deletions overlapping KLHL13. The exonic portions of WDR44 and SLC6A14 each had several overlapping, smaller deletions. Evaluation using DECIPHER (https://decipher. sanger.ac.uk/, last accessed 7/7/15) showed 3 individuals with 46,XY chromosome complements and overlapping deletions. Two individuals had large deletions in the $\mathrm{X}$ chromosome (92.91 and $152.14 \mathrm{Mb}$, respectively), suggesting that the sex chromosome assignment and other cytogenetic findings may not be directly comparable to the patient described in this study. One individual, described as having mild neurocognitive dysfunction and dysmorphic features, had a de novo deletion in chrX: $115,007,250-116,707,313$ (as well as another de novo deletion in chromosome 11), which overlapped SLC6A14 and CXorf61.

We reviewed available literature on the genes within the deletion interval of the X chromosome. SLC6A14 encodes a beta-alanine carrier responsible for the absorption of essential amino acids in the distal gastrointestinal tract [Anderson et al., 2008]. One Finnish genome-wide association study showed a possible link between muta- 
tions in SLC6A14 and obesity [Suviolahti et al., 2003]. KLHL13 belongs to a group of 42 known genes, referred to as the Kelch genes, which encode structurally similar proteins involved in the post-translational ubiquitination process. KLHL13 functions as an adaptor protein that complexes with Cullin 3 and other proteins to form a ubiquitin-protein ligase complex that plays an essential role in cell cycle regulation, particularly during mitotic division and cytokinesis, a process closely linked to ciliogenesis [Sumara et al., 2007]. The functions of many of the Kelch genes are not well understood, although mutations in several genes in this family are known to be involved in human disease. For example, mutations in KLHL7 have been found in individuals with autosomal dominant retinitis pigmentosa and mutations in KLHL6, KLHL19, KLHL20, and KLHL37 have been associated with various cancers [Dhanoa et al., 2013]. There is no published literature connecting KLHL13 mutations to human disease. CXorf61 (also known as CT83) encodes a cancer/testis antigen that interacts with tumor-specific cytotoxic T-cell lymphocytes in lung cancer cells and thought to be a potential immunotherapy target [Caballero and Chen, 2009]. Lastly, a portion of the X chromosome containing WDR44 is deleted in our patient. This gene encodes the protein Rab11BP, which is an effector protein that interacts with a GTP-binding protein, Rab11, and is involved in endosome recycling [Zeng et al., 1999]. Rab11 is one of several GTP-binding proteins in the Rab family involved in intracellular transport and early ciliogenesis [Lim et al., 2011]. There is no published literature describing mutations in WDR44 causing a human phenotype, but this gene belongs to a gene family in which mutations can cause multiple phenotypes with some similarity to that observed in our patient. Mutations in DNAHI1, IFT80, IFT140, WDR19, and WDR35 cause conditions including primary ciliary dyskinesia, asphyxiating thoracic dystrophy, Mainzer-Saldino syndrome, and cranioectodermal dysplasia [Lander et al., 2001; Solomon et al., 2013].

Although there are no available studies of mutations in the described deleted genes and MCA in the newborn period, and these genes have not been clearly shown to encode proteins that are involved in the structure or function of primary cilia, the collective congenital anomalies found in our patient have clinical similarities with disorders of known ciliary dysfunction [Badano et al., 2006]. Cilia are hair-like structures found on the cell surface of almost every cell in the human body. These microtubulebased structures extend from the basal body into the intracellular fluid, and their structure has been highly conserved throughout evolution and across organ systems
[Hildebrandt et al., 2011]. Primary cilia appear to be particularly important in various cell signaling pathways including transmitting extracellular signals and transporting these signals intracellularly using a complex system of intraflagellar transport [Pedersen and Rosenbaum, 2008]. Proteome analysis has identified more than 1,000 ciliaassociated proteins [Drummond, 2012], although the genes encoding these proteins and their specific functions have only been fully elucidated in a small subset. Given the widespread function of primary cilia, genetic alterations affecting any of the hundreds of proteins that constitute primary cilia can result in a frequently recognizable pattern of multiple organ dysfunction [Hildebrandt et al., 2011]. Recently, the term 'ciliopathy' has been used to describe a certain clinical phenotype that includes multiple disorders resulting from defects in ciliary structure or function [Badano et al., 2006].

Our patient's renal U/S identified small cortical cysts, a characteristic ciliopathy feature. Primary cilia located in the renal epithelium play a role in sensing environmental signals involved in cell differentiation and proliferation [Yoder et al., 2002]. Defects in cilia located in the collecting ducts of renal epithelium, as seen in polycystic kidney disease, cause abnormal cell differentiation resulting in renal cysts [Nauli et al., 2003]. Renal cysts have also been described in more complex, genetically heterogeneous disorders that affect multiple organ systems. Bardet-Biedl syndrome, an autosomal recessive disorder caused by defects in an elaborate protein complex involved in signaling receptor trafficking to and from the cilia, is characterized by renal cysts, polydactyly, retinitis pigmentosa, truncal obesity, and intellectual disability [Scheidecker et al., 2014]. Renal cysts can also be found in other multisystem ciliopathies including Joubert syndrome [Valente et al., 2013] and Meckel-Gruber syndrome [Badano et al., 2006].

The patient was also found to have several skeletal and related anomalies including a bell-shaped chest, dysplastic hips, preaxial polydactyly, and talipes equinovarus. These clinical features resemble skeletal findings seen in multisystem ciliopathies with skeletal dysplasia as a cardinal feature such as Jeune syndrome, a ciliopathy characterized by a severely constricted bell-shaped chest, short-limbed short stature and polydactyly in some patients. The prognosis of patients with Jeune syndrome depends on the degree of restriction of the chest cavity as it relates to lung development. Those who survive past the neonatal period have been found to have renal cysts, retinal degeneration and liver disease. Jeune syndrome is a genetically heterogenous disorder caused by mutations in
Pavey/Vilboux/Babcock/Ahronovich/ Solomon 
at least 4 different genes known to encode proteins involved in intraflagellar transport [Huber and CormierDaire, 2012]. The role of primary cilia in normal skeletal development may be explained by the presence of cilia in ectodermal and mesenchymal cells in the limb bud [Haycraft et al., 2005; Badano et al., 2006]. Ciliary dysfunction can have profound effects on limb development, again potentially explaining the skeletal anomalies seen in this patient.

CNS abnormalities such as agenesis of the corpus callosum, neural tube defects, and DWMs have been reported in disorders of cilia structure and function [Badano et al., 2006]. Head U/S on our patient revealed an abnormal posterior fossa, suggestive of a variant of DWM. The mechanism of ciliary dysfunction leading to CNS malformations is not well understood, and DWM can occur in patients without ciliopathies. Recent research focused on ciliary function in early embryogenesis revealed that signaling mechanisms involved in cell migration, polarization, and differentiation during neural tube formation can been linked to cilia [Barker et al., 2014]. Ciliopathies with CNS abnormalities include Senior-Loken syndrome, Joubert syndrome and Meckel-Gruber syndrome [Badano et al., 2006].

The presence of remote cis-acting enhancers or silencers in the region affecting the expression of one or more genes flanking the deletion cannot be excluded. DOCK11 and AGTR2 are adjacent genes that could be affected by such regulatory elements (fig 1). DOCK11 is a guanine nucleotide exchange factor for $\mathrm{Cdc} 42$, a protein critically important in cell cycle regulation and cilium biogenesis [Lin et al., 2006]. While mutations in DOCK11 have not been linked to human disease, mutations in DOCK6 are one cause of Adams-Oliver syndrome [Shaheen et al., 2011], a congenital condition characterized by aplasia cutis congenita and terminal transverse limb defects. AGTR2 encodes angiotensin receptor 2 , which is part of the renin-angiotensin-aldosterone system (RAAS) and is involved in the regulation of blood pressure and water and electrolyte balance. Hypertension related to variations in the RAAS pathway is implicated in end-stage renal disease in patients with polycystic kidney disease [Ramanathan et al., 2014]. Variants in AGTR2 have also been associated with ureteropelvic junction stenosis or atresia and various other nephropathic disorders [Nishimura et al., 1999].

In summary, we present a male patient with MCA and a 1.9-Mb deletion in the $\mathrm{X}$ chromosome. The deleted region in the $\mathrm{X}$ chromosome contains 4 incompletely described genes. We postulate that one or more of these genes may be related to the structure or function of the primary cilia, causing the deletion to result in lethal congenital anomalies affecting multiple organ systems and resembling previously described ciliopathies. These genes should be considered candidate genes for further molecular studies in patients with similar phenotypes.

This report is limited by lack of complete clinical information due to the severity of the condition as well as nonavailability of the patient and family for further biological samples for genetic and functional testing that may provide further evidence for or against this deletion as causing the observed phenotype. In the absence of tissue samples from our patient and his family members, an animal or other model organism could provide further insight into the expression and function of these genes as well as the result of their dysregulation.

\section{Acknowledgments}

This work was supported by the Inova Translational Medicine Institute (ITMI), Inova Fairfax Hospital, Falls Church, Va., USA. This study makes use of data generated by the DECIPHER Consortium. A full list of centers that contributed to the generation of the data is available at http://decipher.sanger.ac.uk/. Funding for the DECIPHER project was provided by the Wellcome Trust. The authors would like to express their gratitude to the patient and family described.

\section{Disclaimer}

The views expressed in this article are those of the authors and do not necessarily reflect the official policy or position of the Department of the Army, Department of Defense, or the US Government. The identification of specific products or scientific instrumentation does not constitute endorsement or implied endorsement on the part of the author, Department of Defense, or any company agency. While we generally excise references to products, companies, manufacturers, organizations, etc. in government produced works, the abstracts produced and other similarly situated researcher presents a special circumstance when such product inclusions become an integral part of the scientific endeavor.

\section{Statement of Ethics}

This study was approved by the Inova Health System Institutional Review Board.

\section{Disclosure Statement}

The authors have no conflicts of interest to declare. 


\section{References}

Anderson C, Ganapathy V, Thwaites D: Human solute carrier SLC6A14 is the beta-alanine carrier. J Physiol 586:4061-4067 (2008).

Badano J, Mitsuma N, Beales P, Katsanis N: The ciliopathies: an emerging class of human genetic disorders. Annu Rev Genomics Hum Genet 7:125-148 (2006).

-Barker A, Thomas R, Dawe H: Meckel-Gruber syndrome and the role of primary cilia in kidney, skeleton, and central nervous system development. Organogenesis 10:96-107 (2014).

-Biesecker L, Green R: Diagnostic clinical genome and exome sequencing. N Engl J Med 370: 2418-2425 (2014).

Caballero OL, Chen YT: Cancer/testis (CT) antigens: potential targets for immunotherapy. Cancer Sci 100:2014-2021 (2009).

Dhanoa B, Cogliati T, Satish A, Bruford E, Friedman J: Update on the Kelch-like (KLHL) gene family. Hum Genomics 7:13 (2013).

Drummond IA: Cilia functions in development. Curr Opin Cell Biol 24:24-30 (2012).

-Haycraft C, Banizs B, Aydin-Son Y, Zhang Q, Michaud E, Yoder B: Gli2 and Gli3 localize to cilia and require the intraflagellar transport protein polaris for processing and function. PLoS Genet 1:e53 (2005).

Hildebrandt F, Benzing T, Katsanis N: Ciliopathies. N Engl J Med 364:1533-1543 (2011).

-Huber C, Cormier-Daire V: Ciliary disorder of the skeleton. Am J Med Genet C Semin Med Genet 160C:165-174 (2012).

-Lander E, Linton LM, Birren B, Nusbaum C, Zody $\mathrm{MC}$, et al: Initial sequencing and analysis of the human genome. Nature 409:860-921 (2001).

- Lim Y, Chua C, Tang B: Rabs and other small GTPases in ciliary transport. Biol Cell 103: 209-221 (2011).

Lin Q, Yang W, Baird D, Feng Q, Cerione RA: Identification of a DOCK180-related guanine nucleotide exchange factor that is capable of mediating a positive feedback activation of Cdc42. J Biol Chem 281:35253-35262 (2006).
Lu X, Phung MT, Shaw C, Pham K, Neil S, et al: Genomic imbalances in neonates with birth defects: high detection rates by using chromosomal microarray analysis. Pediatrics 122 : 1310-1318 (2008).

Majewski J, Schwartzentruber J, Lalonde E, Montpetit A, Jabado N: What can exome sequencing do for you? J Med Genet 48:580589 (2011).

Marian A: Challenges in medical applications of whole exome/genome sequencing discoveries. Trends Cardiovasc Med 22:219-223 (2012).

Miller D, Adam M, Aradhya S, Biesecker L, Brothman A, et al: Consensus statement: chromosomal microarray is a first-tier clinical diagnostic test for individuals with developmental disabilities or congenital anomalies. Am J Hum Genet 86:749-764 (2010).

Nauli S, Alenghat F, Luo Y, Williams E, Vassilev $\mathrm{P}$, et al: Polycystins 1 and 2 mediate mechanosensation in the primary cilium of kidney cells. Nat Genet 33:129-137 (2003).

Nishimura H, Yerkes E, Hohnefellner K, Miyazaki Y, Ma J, et al: Role of the angiotensin type 2 receptor gene in congenital anomalies of the kidney and urinary tract, CAKUT, of mice and men. Mol Cell 3:1-10 (1999).

Pedersen L, Rosenbaum J: Intraflagellar transport (IFT) role in ciliary assembly, resorption and signalling. Curr Top Dev Biol 85:23-61 (2008).

Ramanathan G, Elumala R, Periyasamy S, Lakkakula B: Role of renin-angiotensin-aldosterone system gene polymorphisms and hypertension-induced end-stage renal disease in autosomal dominant polycystic kidney disease. Iran J Kidney Dis 8:265-277 (2014).

Resta N, Memo L: Chromosomal microarray (CMA) analysis in infants with congenital anomalies: when is it really helpful? J Matern Fetal Neonatal Med 25 Suppl 4:124-126 (2012).
Scheidecker S, Etard C, Pierce N, Geoffroy V, Schaeffer E, et al: Exome sequencing of Bardet-Biedl syndrome patient identifies a null mutation in the BBSome subunit $B B I P 1$ (BBS18). J Med Genet 51:132-136 (2014).

Shaheen R, Faqeih E, Sunker A, Morsy H, AlSheddi $\mathrm{T}$, et al: Recessive mutations in DOCK6, encoding the guanidine nucleotide exchange factor DOCK6, lead to abnormal actin cytoskeleton organization and AdamsOliver syndrome. Am J Hum Genet 89:328333 (2011).

Solomon B, Nguyen A, Bear K, Wolfsberg T: Clinical Genomic Database. Proc Natl Acad Sci 110:9851-9855 (2013).

Sumara I, Quadroni M, Frei C, Olma M, Sumara $\mathrm{G}$, et al: A Cul3-based E3 ligase removes Aurora B from mitotic chromosomes, regulating mitotic progression and completion of cytokinesis in human cells. Dev Cell 12:887-900 (2007).

Suviolahti E, Oksanen L, Ohman M, Cantor R, Ridderstrale $\mathrm{M}$, et al: The SLC6A14 gene shows evidence of association with obesity. J Clin Invest 112:1762-1772 (2003).

Valente E, Dallapiccola B, Bertini E: Joubert syndrome and related disorders. Handb Clin Neurol 113:1879-1888 (2013).

Volk A, Conboy E, Wical B, Patterson M, Kirmani S: Whole-exome sequencing in the clinic: lessons from six consecutive cases from the clinician's perspective. Mol Syndromol 6:23-31 (2015).

Yoder B, Hou X, Guay-Woodford L: The polycystic kidney disease proteins, polycystin-1, polycystin-2, polaris, and cystin, are co-localized in renal cilia. J Am Soc Nephrol 13:25082516 (2002).

Zeng J, Ren M, Gravotta D, De Lemos-Chiarandini C, Lui M, et al: Identification of a putative effector protein for rab11 that participates in transferrin recycling. Proc Natl Acad Sci USA 96:2840-2845 (1999). 